\title{
Outcomes of Extracorporeal Membrane Oxygenation in Influenza vs. COVID-19 During the First Wave of COVID-19
}

\author{
Cameron Blazoski ${ }^{1}$, Michael Baram $^{1}$, and Hitoshi Hirose ${ }^{1}$ \\ ${ }^{1}$ Thomas Jefferson University Hospital
}

May 29, 2021

\begin{abstract}
Purpose: Extracorporeal membrane oxygenation (ECMO) is a refractory treatment for acute respiratory distress syndrome (ARDS) due to influenza and severe acute respiratory syndrome coronavirus 2 (SARS-CoV-2, also referred to as COVID-19). We conducted this study to compare the outcomes of influenza patients treated with veno-venous-ECMO (VV-ECMO) to COVID-19 patients treated with VV-ECMO, during the first wave of COVID-19. Materials and Methods: Patients in our institution with ARDS due to COVID-19 or influenza who were placed on ECMO between August 1, 2010 and September 15, 2020 were included in this comparative, retrospective study. To improve homogeneity, only VV -ECMO patients were analyzed. The clinical characteristics and outcomes were extracted and analyzed. Results: 28 COVID-19 patients and 17 influenza patients were identified and included. ECMO survival rates were 68\% (19/28) in COVID-19 patients and 94\% (16/17) in influenza patients $(\mathrm{p}=0.04)$. 30-day survival rates after ECMO decannulation were $54 \%(15 / 28)$ in COVID-19 patients and $76 \%(13 / 17)$ in influenza patients $(\mathrm{p}=0.13)$. COVID-19 patients spent a longer time on ECMO compared to flu patients (21 days vs. 12 days, $\mathrm{p}=0.025)$, and more COVID-19 patients (26/28 vs. $2 / 17)$ were on immunomodulatory therapy prior to ECMO initiation $(\mathrm{p}<0.001)$. COVID-19 patients had higher rates of new infections during ECMO (50\% vs. $18 \%$, p=0.03) and bacterial pneumonia ( $36 \%$ vs $6 \%, \mathrm{p}=0.024)$. Conclusions: COVID-19 patients who were treated in our institution with VV-ECMO had statistically lower ECMO survival rates than influenza patients. It is possible that COVID-19 immunomodulation therapies may increase the risk of other superimposed infections.
\end{abstract}

Outcomes of Extracorporeal Membrane Oxygenation in Influenza vs. COVID-19 During the First Wave of COVID-19.

Cameron M. Blazoski, BA, ${ }^{1}$ Michael Baram, MD ${ }^{2}$ Hitoshi Hirose, MD, PhD. ${ }^{3}$

${ }^{1}$ Sidney Kimmel Medical College, Thomas Jefferson University, Philadelphia, PA

${ }^{2}$ Division of Pulmonary and Critical Care, Thomas Jefferson University, Philadelphia, PA.

${ }^{3}$ Department of Surgery, Thomas Jefferson University, Philadelphia, PA

Running title: ECMO Influenza vs. COVID-19

Address for correspondence:

Hitoshi Hirose, MD.

Dept. Surgery, Thomas Jefferson University

1025 Walnut Street, Suite 605, Philadelphia, PA 19107, USA.

Tel: 215-955-5654, Fax: 215-955-6010, Email: Hitoshi.Hirose@jefferson.edu

Word count: 2735 
Conflict of Interests: None declared

Financial support: None.

Data: The data that support the findings of this study are available from the corresponding author upon reasonable request

\section{Abstract}

Purpose: Extracorporeal membrane oxygenation (ECMO) is a refractory treatment for acute respiratory distress syndrome (ARDS) due to influenza and severe acute respiratory syndrome coronavirus 2 (SARSCoV-2, also referred to as COVID-19). We conducted this study to compare the outcomes of influenza patients treated with veno-venous-ECMO (VV-ECMO) to COVID-19 patients treated with VV-ECMO, during the first wave of COVID-19.

Materials and Methods: Patients in our institution with ARDS due to COVID-19 or influenza who were placed on ECMO between August 1, 2010 and September 15, 2020 were included in this comparative, retrospective study. To improve homogeneity, only VV -ECMO patients were analyzed. The clinical characteristics and outcomes were extracted and analyzed.

Results : 28 COVID-19 patients and 17 influenza patients were identified and included. ECMO survival rates were $68 \%(19 / 28)$ in COVID-19 patients and $94 \%(16 / 17)$ in influenza patients $(\mathrm{p}=0.04)$. 30-day survival rates after ECMO decannulation were 54\% (15/28) in COVID-19 patients and $76 \%(13 / 17)$ in influenza patients $(\mathrm{p}=0.13)$. COVID-19 patients spent a longer time on ECMO compared to flu patients (21 days vs. 12 days, $\mathrm{p}=0.025)$, and more COVID-19 patients $(26 / 28$ vs. $2 / 17$ ) were on immunomodulatory therapy prior to ECMO initiation $(\mathrm{p}<0.001)$. COVID-19 patients had higher rates of new infections during ECMO ( $50 \%$ vs. $18 \%, \mathrm{p}=0.03)$ and bacterial pneumonia ( $36 \%$ vs $6 \%, \mathrm{p}=0.024)$.

Conclusions : COVID-19 patients who were treated in our institution with VV-ECMO had statistically lower ECMO survival rates than influenza patients. It is possible that COVID-19 immunomodulation therapies may increase the risk of other superimposed infections.

\section{(Word count of abstract: 247)}

\section{Introduction}

One of the main concerns of coronavirus disease 2019 (COVID-19) infection is development of acute respiratory distress syndrome (ARDS). Although the definition of ARDS has changed over the decades, its clinical context remains the same: a rapidly progressive inflammatory syndrome that impairs oxygen transport in the lungs. ${ }^{1-3}$ Historically, the most common viral cause of adult ARDS prior to COVID-19 infection was influenza pneumonia, and the complication of ARDS from influenza is known to be associated with an increased mortality. ${ }^{4,5}$ The pulmonary injury in ARDS due to COVID-19 has been shown to resemble other viral causes of ARDS, and as expected, the severity of ARDS is associated with significantly worsened mortality among COVID-19 patients. ${ }^{1,6,7}$

Due to the high mortality rate of ARDS due to COVID-19, there has been a high demand for refractory treatment options in patients who do not improve upon standard ventilation and treatment. Thus, extracorporeal membrane oxygenation (ECMO) was used in select cases of COVID-19 with refractory ARDS and severe hypoxemia. ${ }^{7-10}$ ECMO is a temporary form of mechanical cardiopulmonary support, used in patients with severe cardiac and/or respiratory shock. ECMO was first clinically used in 1972 and has been increasingly incorporated into standard practice in the past two decades. ${ }^{11-13}$ Despite controversial and conflicting evidence on its overall efficacy, ECMO has become a common treatment for patients with refractory ARDS. ${ }^{13-16}$

In cases of influenza, ECMO can be used adjunctive support in cases complicated by severe ARDS. ${ }^{17}$ With knowledge learned from years of critical care and development of protocols, there is a good understanding 
of how to properly care for patients with influenza. ${ }^{18,19}$ It is known that in the setting of influenza, immunosuppression with steroids increases the duration of viral shedding and worsens mortality, ${ }^{20}$ so high dose immunosuppression is usually avoided. If traditional therapies fail to stabilize the patient, early initiation of ECMO in critically ill patients with influenza can improve their chance of survival by promoting lung protective ventilator strategies without compromising required gas exchange. The amount of benefit provided by ECMO in complement to anti-influenza agents is unclear, though outcomes have been acceptable. ${ }^{17}$ In contrast, we have not identified a specific, proven treatment protocol for COVID-19 infection despite the widespread use of supportive measures such as remdesivir, lung-protective ventilator strategies, antiinflammatory agents, and steroids. ${ }^{21}$ Despite initial support for some of these agents, some subsequent research has been less optimistic. ${ }^{21,22}$

While ARDS due to COVID-19 has been compared to ARDS caused by influenza, severe cases of COVID-19 continue to demonstrate high mortality rates, and the similarities and differences between the two diseases are not well understood. Similarly, despite recent studies on the use of ECMO in COVID-19 patients, ${ }^{23-25}$ there remains a lack of evidence documenting the overall efficacy of ECMO in treating ARDS due to COVID19. This paper will compare the outcomes and efficacy of ECMO in treating patients with ARDS due to COVID-19 or influenza to better understand the prognosis of ARDS due to COVID-19 and the use of ECMO in treating it.

\section{Materials and Methods}

Patients were identified within an IRB-approved, prospectively maintained ECMO database (IRB approval \# 11D.185) at our institution from August 1, 2010 to September 15, 2020. Patients who were confirmed to have influenza or COVID-19 who underwent ECMO were included in this study. Data from these patients was retrospectively extracted and details were further studied by reviewing medical records. Inclusion criteria included a positive COVID-19 test and a diagnosis of ARDS. ECMO placement was determined by a multidisciplinary team that included a cardiac surgeon, a pulmonary-critical care physician, and a cardiovascular intensivist.

The indications for ECMO placement were the same as those listed in our previous paper, ${ }^{26}$ and Table 1 includes the list of contraindications for ECMO placement in COVID-19 patients. The exclusion criteria for COVID-19 patients may be more restrictive than in non-COVID-19 patients, due to the limited resources available during the first wave of the pandemic and challenges due to increased isolation needs.

During the first wave, our institution did not utilize veno-arterial ECMO (VA-ECMO) in patients with COVID-19, due to limited resources and an unclear understanding of the reversibility of the disease. In influenza patients, 7 patients were placed for VA-ECMO for cardiac dysfunction. However, these VA-ECMO patients were excluded from this study to ensure the appropriate comparisons.

Due to resource allocation and isolation concerns, COVID-19 and influenza patients were treated differently. We traditionally used single double-lumen cannula (Avalon ${ }^{\circledR}$ cannula, Avalon Laboratories, Rancho Dominguez, CA) for VV- ECMO patients, but this had to be modified for COVID-19. In COVID-19 patients, VV-ECMO was placed using the femoral and internal jugular veins (Figure 1). This change in insertion practice did not result in procedural complications, but it did affect body mass index (BMI) restrictions. All cannulation was performed in the ICU without transport to either the operating room or catheterization lab unless an issue occurred during the bedside cannulation. Since single dual lumen ECMO cannula placement always requires fluoroscopy and echocardiography, which requires additional personnel including radiology technicians and an echocardiography technician, the utilization of the Avalon ${ }^{\circledR}$ cannula was discouraged. ${ }^{26}$

Due to the COVID-19 pandemic, our institution did not offer a mobile ECMO program outside of our hospital network to avoid possible exposure of required personnel including the ECMO surgeon, perfusionist, and transfer nurses at the local site. Instead of activating mobile ECMO cannulation teams, we encouraged local cardiac surgeons to place ECMO at their institutions and then transport the patient to our facility.

The general management of ECMO has been described in one of our prior papers. ${ }^{28,29}$ Briefly, after placement 
of ECMO, the ventilator was set to the ARDSnet protocol. ${ }^{18}$ The typical setting was pressure controlled ventilation, rate 15 per minute, PEEP $15 \mathrm{~cm} \mathrm{H}_{2} \mathrm{O}$, delta $\mathrm{P} 15 \mathrm{~cm} \mathrm{H}_{2} \mathrm{O}$, and inspiratory time 1.5 seconds until recovery of the respiratory function. ${ }^{30}$ Paralytics were discontinued within 24 hours of ECMO initiation, unless ventilatory desynchrony resulted in hemodynamic instability. Sedatives were used to achieve a RAS score of negative 1-2. Blood pressure was maintained at a mean arterial pressure of at least $60 \mathrm{~mm} \mathrm{Hg}$ with vasopressors and/or fluid as appropriate. A heparin drip was started once PTT fell below 50 seconds after cannulation and maintained at an anti-Xa level of 0.3-0.5 IU/ml. If bleeding complications were observed, the anticoagulation was held and then restarted at a lower anti-Xa goal of 0.1-0.3 IU/ml.

Timing of the decannulation was determined by chest x-ray findings, lung mechanics, and gas exchange. Before decannulation, the sweep gas was discontinued for at least 24 hours to ensure the lungs were able to exchange oxygen and carbon dioxide appropriately. For COVID-19 cases, we encouraged bed-side decannulation and discouraged transporting to operating room to limit exposure to COVID-19.

For our primary comparison, all adult patients who met our inclusion criteria were divided by their cause of ARDS, either influenza or COVID-19. The baseline characteristics, clinical characteristics, and outcomes were calculated and compared between the two groups. The primary endpoints of this study were ECMO survival and 30-day survival. ECMO survival was defined as surviving at least 24 hours post decannulation.

Data was expressed as the number with percentage, mean +/- standard deviation, or median (quantile) as appropriate. The two groups were compared using chi-squared tests for categorical variables and standard t-tests for continuous variables as appropriate, with significance accepted at a P-value less than 0.05.

\section{Results}

45 patients with ARDS who underwent VV-ECMO placement met our inclusion criteria and were included in this study. Of those patients, 28 had ARDS due to COVID-19 and 17 had ARDS due to influenza. $64 \%$ of COVID-19 patients $(n=18)$ and $65 \%$ of influenza patients $(n=11)$ were transferred from an outside of hospital to our institution, with a significantly higher percentage of COVID-19 patients having ECMO initiated outside of our hospital ( $50 \%$ vs. $12 \%, \mathrm{p}=0.01)$. Avalon cannula ${ }^{\odot}$ was used more often in influenza patients than COVID-19 patients ( $88 \%$ vs. $7 \%, \mathrm{p}<0.01$ ). The average duration of ECMO utilization in COVID-19 patients was 21.4 days, which was significantly longer than the average duration of influenza patients (12.2 days) $(\mathrm{p}=0.03)$.

COVID-19 patients had lower incidence of pre-ECMO comorbidities including history of coronary artery disease $(\mathrm{p}=0.02)$ and acute kidney injury $(\mathrm{p}=0.05)$. They also had a lower body surface area $(\mathrm{p}=0.04)$. There were no statistically significant differences in the vital signs before ECMO placement. Patient demographics and pre-ECMO characteristics are displayed in Table 2.

Patients with ARDS due to COVID-19 had a significantly decreased ECMO survival rate ( $\mathrm{p}=0.04)$. Of the COVID-19 patients, 19 (68\%) survived ECMO and 15 (54\%) survived to 30 days after decannulation. Among influenza patients, 16 (94\%) survived ECMO and $13(76 \%)$ survived to 30 days after decannulation.

The most common complication among COVID-19 patients was the development of a new infection during ECMO, with 14 patients (50\%) developing a new infection after the placement of ECMO. Among influenza patients, the most common complications were renal failure, GI bleeds, and new infections, all of which occurred in 3 patients (18\%). COVID-19 patients had significantly higher rates of bacterial pneumonia $(\mathrm{p}=0.03)$, any new infections $(\mathrm{p}=0.03)$, and blood culture-positive sepsis $(\mathrm{p}=0.04)$, as displayed in Table 3 .

\section{Discussion}

The primary findings of this study relate to the survival rate and complications associated with patients with ARDS due to COVID-19 who are treated with VV-ECMO. Patients with ARDS due to COVID-19, compared to patients with ARDS due to influenza, had a significantly higher ECMO mortality, with less patients surviving to ECMO decannulation. At the same time, patients with COVID-19 had an increased 
risk for developing new infections, with significantly higher rates of pneumonia and sepsis among COVID-19 patients.

The treatment of ARDS with ECMO remains disputed, despite its increased use in treating ARDS in the past decade. ${ }^{13,14}$ While the exact mortality rate of treating ARDS with ECMO varies by research study, it is typically accepted to range between $34-39 \% .{ }^{13,15,16}$ This mortality rate is still better on ECMO than was reported by the ARDS definition task force, which highlights the importance of patient selection. ${ }^{3}$ Thus, it is generally recognized that ECMO should be primarily used for refractory cases of ARDS, in which a patient remains severely hypoxic despite aggressive treatment. ${ }^{15}$

A 2020 study by Acosta and Singer suggests that the pathogenesis and clinical course of ARDS due to influenza resembles ARDS due to COVID-19. ${ }^{32}$ However, differences in patient outcomes illustrates that there must be an difference between the two. The paper speculated that these severe cases may be due to SARSCoV-2's ability to dampen the inflammatory response to severe infection and impede normal pulmonary recovery to damage, which would exacerbate ARDS and lead to the observed increased mortality rate among patients with COVID-19. This again brings to question the treatment protocols that utilize potent and high dose immunosuppressants. ${ }^{21,22}$

Acosta and Singer emphasize that while mild to moderate cases of influenza and COVID-19 may present similarly, their prognoses diverge in severe cases. This idea is supported by a body of evidence which indicate that severe cases of COVID-19 have a higher mortality rate than severe cases of influenza. For example, one study found that patients who are admitted to the ICU for COVID-19 have a 3.7 times higher risk of death than patients treated in the ICU for influenza. ${ }^{6}$ Similarly, another study detailed that hospitalized patients in the Veteran's Health Administration had a 5 times higher risk for death than hospitalized patients with influenza. ${ }^{33}$ These studies are consistent with the trends at our institution.

In our study, we also discovered that while COVID-19 patients had lower rates of certain pre-existing conditions, they still had a higher mortality rate. Influenza patients had significantly higher rates of pre-ECMO acute kidney injury and coronary artery disease, as well as a higher body surface area. However, this is likely due to our restricted inclusion criteria for ECMO placement in COVID-19 patients and should not be used to make any definite conclusions on the impact of pre-existing conditions on mortality rate between influenza and COVID-19 patients.

An important finding of our study was the significantly higher rates of secondary bacterial infection in COVID-19 patients. Of the 28 COVID-19 patients, 14 (50\%) developed a new infection during ECMO placement, with $10(36 \%)$ developing bacterial pneumonia and $9(32 \%)$ developing blood culture-positive sepsis. It is important to note that bacterial infection is a common complication for any patient who undergoes ECMO placement, and infections can lead to pneumonia and sepsis, both of which significantly increase these patients' mortality rate. ${ }^{11}$ However, our research indicates that comparatively, patients with ARDS due to COVID-19 develop new infections more commonly when on ECMO than patients with ARDS due to influenza. This finding is backed by recent research on cases of COVID-19. COVID-19 has been statistically linked with cases of bacterial superinfection, especially in critically ill patients, which has been documented to lead to bacterial pneumonia and sepsis. ${ }^{34-36}$ Secondary bacterial infection in COVID-19 patients has also been significantly associated with poor outcomes and an increased mortality rate, even when patients are treated with aggressive antimicrobial therapies. ${ }^{37}$

The increased risk of infection may be a result of immunomodulation therapy in treating COVID-19. While immunomodulation therapy has been shown to decrease the mortality rate of COVID-19, ${ }^{38,39}$ it has also been associated with an increased infection rate. In our study, 93\% of COVID-19 patients were on some form of immunotherapy - either steroids, interleukin inhibitors, or both - while only $12 \%$ of influenza patients were immunosuppressed prior to ECMO initiation $(\mathrm{p}<0.001)$. Ultimately, it is possible - and even likely - that the decreased ECMO survival rate among our COVID-19 patients is partially caused by the increased incidence of bacterial superinfections.

Our study is limited by its small sample size and being based in one hospital center that provided ECMO 
support for a twelve hospital health system. It is also possible that there was selection bias in this study, even though ECMO placement was determined by a multidisciplinary team of physicians. Moreover, influenza patients dated back to 2010, while all COVID-19 patients were treated in 2020; it is possible that changes in ECMO protocol due to COVID-19 and associated treatment protocols with ECMO could impact patient outcomes and complication rates.

Despite its limitations, this study provides significant data on 28 patients with COVID-19 and effectively compares patients with ARDS due to COVID-19 to patients with ARDS due to influenza. This paper is one of a growing number of studies on COVID-19, and we hope that our findings contribute to a better understanding of how to effectively treat COVID-19.

\section{Conclusion}

Based on our results, we conclude that there are significant differences in the use of VV-ECMO in treating ARDS due to COVID-19 to treating ARDS due to influenza. COVID-19 patients appear to be at a higher risk of bacterial superinfection, and prevention and control of bacterial infections may be critical in improving survival. More research is needed to understand the efficacy and risks of using ECMO to treat cases of COVID-19.

Table 1 : Contraindications for ECMO in COVID-19

\begin{tabular}{l}
\hline Standard contraindications \\
\hline Age $>70$ y.o. \\
Body mass index $>45$ with high risk of vascular access \\
Mechanical ventilation $>7$ days \\
Multiorgan failure \\
End stage liver disease \\
Irreversible neurological damage \\
Contraindications of anticoagulation \\
Cardiac arrest without ROSC \\
Relative contraindications \\
Age $>65$ y.o. \\
Body mass index $>35$ \\
Mechanical ventilation $>5$ days \\
Active bacterial blood stream infection \\
Severe COPD Cirrhosis Chronic heart failure \\
Inability of access neuro status \\
High lactate related to low perfusion status \\
Limited activity at home \\
No family or appropriate power of attorney Outside of institutional network \\
\hline
\end{tabular}

Table 2 : Demographics and baseline characteristics of studied patients. Data is expressed with number (percentage) or mean \pm standard deviation.

\begin{tabular}{lllll}
\hline & All patients & Influenza & COVID-19 & \\
\hline $\begin{array}{l}\text { Number of patients } \\
\text { Characteristics }\end{array}$ & $\mathrm{n}=45$ & $\mathrm{n}=17$ & $\mathrm{n}=28$ & P-value \\
Age (years) & $51.9 \pm 11.2$ & $49.5 \pm 13.2$ & $53.3 \pm 9.8$ & 0.314 \\
Male & $28(62 \%)$ & $9(53 \%)$ & $19(68 \%)$ & 0.317 \\
Body surface area $\left(\mathrm{cm}^{2}\right)$ & $2.11 \pm 0.27$ & $2.24 \pm 0.27$ & $2.04 \pm 0.35$ & 0.036 \\
Body mass index & $34.95 \pm 7.82$ & $37.58 \pm 9.06$ & $33.36 \pm 6.63$ & 0.107 \\
Underlying Conditions & & & &
\end{tabular}




\begin{tabular}{lllll}
\hline & All patients & Influenza & COVID-19 & \\
\hline Pre-ECMO positive blood culture & $10(22 \%)$ & $4(24 \%)$ & $6(0 \%)$ & 0.869 \\
Smoking & $10(22 \%)$ & $5(29 \%)$ & $5(18 \%)$ & 0.366 \\
Coronary artery disease & $3(7 \%)$ & $3(18 \%)$ & $0(0 \%)$ & 0.021 \\
Chronic lung disease & $5(11 \%)$ & $3(18 \%)$ & $2(7 \%)$ & 0.277 \\
Diabetes & $13(29 \%)$ & $5(29 \%)$ & $8(29 \%)$ & 0.952 \\
Pre-ECMO acute renal injury & $18(40 \%)$ & $10(59 \%)$ & $8(29 \%)$ & 0.045 \\
Pre-ECMO Immunotherapy & $28(62 \%)$ & $2(12 \%)$ & $26(93 \%)$ & $<0.001$ \\
Pre-ECMO Vital Signs & & & & \\
Temperature $\left({ }^{\circ}\right.$ F) & $99.7 \pm 1.8$ & $100.1 \pm 2.0$ & $99.04 \pm 1.6$ & 0.074 \\
Heart Rate & $107.2 \pm 25.5$ & $115.8 \pm 26.5$ & $102.0 \pm 23.9$ & 0.089 \\
Respiratory rate & $27.4 \pm 4.7$ & $26.9 \pm 4.6$ & $27.7 \pm 4.8$ & 0.581 \\
Mean arterial pressure (mm Hg) & $82.3 \pm 15.7$ & $78.1 \pm 12.8$ & $84.9 \pm 15.1$ & 0.115 \\
FiO2 (\%) & $95.3 \pm 13.1$ & $96.6 \pm 14.6$ & $94.6 \pm 12.3$ & 0.640 \\
PEEP (cm) & $15.2 \pm 5.4$ & $15.6 \pm 6.8$ & $15.0 \pm 4.6$ & 0.750 \\
Other & & & & \\
Transfer from outside hospital & $29(64 \%)$ & $11(65 \%)$ & $18(64 \%)$ & 0.977 \\
ECMO initiated other than our hospital & $16(36 \%)$ & $2(12 \%)$ & $14(50 \%)$ & 0.009 \\
Use of Avalon cannula & $15(38 \%)$ & $15(88 \%)$ & $2(7 \%)$ & $<0.001$ \\
\hline
\end{tabular}

Table 3 : Rates of ECMO complications. Data is expressed with number (percentage).

\begin{tabular}{lllll}
\hline & All patients & Influenza & COVID-19 & \\
\hline Number of patients & $\mathrm{n}=45$ & $\mathrm{n}=17$ & $\mathrm{n}=28$ & P-value \\
Length on ECMO (days) & $17.8 \pm 16.1$ & $12.2 \pm 5.7$ & $21.4 \pm 19.4$ & 0.025 \\
ECMO survival & $35(78 \%)$ & $16(94 \%)$ & $19(68 \%)$ & 0.040 \\
30 days after decannulation survival & $28(62 \%)$ & $13(76 \%)$ & $15(54 \%)$ & 0.130 \\
Complications & & & & \\
Renal failure & $10(22 \%)$ & $3(18 \%)$ & $7(25 \%)$ & 0.565 \\
Liver failure & $3(7 \%)$ & $1(6 \%)$ & $2(7 \%)$ & 0.869 \\
Stroke & $2(4 \%)$ & $1(6 \%)$ & $1(4 \%)$ & 0.715 \\
Brain bleed & $2(4 \%)$ & $1(6 \%)$ & $1(4 \%)$ & 0.715 \\
Cannula site bleed & $5(11 \%)$ & $1(6 \%)$ & $4(14 \%)$ & 0.384 \\
GI bleed & $9(20 \%)$ & $3(18 \%)$ & $6(21 \%)$ & 0.758 \\
Any new infection during ECMO & $17(38 \%)$ & $3(18 \%)$ & $14(50 \%)$ & 0.030 \\
Bacterial pneumonia & $11(24 \%)$ & $1(6 \%)$ & $10(36 \%)$ & 0.024 \\
Blood culture-positive sepsis & $10(22 \%)$ & $1(6 \%)$ & $9(32 \%)$ & 0.040 \\
\hline
\end{tabular}

\section{Ledends of Figure}

Figure 1 : Typical veno-venous cannulation in COVID-19 case. Patients were primarily cannulated via the right internal jugular vein and right femoral vein due to anatomical preference.

\section{References}

1. Yang $\mathrm{X}, \mathrm{Yu} \mathrm{Y}, \mathrm{Xu}$ J, et al. Clinical course and outcomes of critically ill patients with SARS-CoV-2 pneumonia in Wuhan, China: a single-centered, retrospective, observational study. The Lancet Respiratory Medicine . 2020;8(5):475-481. doi:10.1016/S2213-2600(20)30079-5

2. Gibson PG, Qin L, Puah SH. COVID-19 acute respiratory distress syndrome (ARDS): clinical fea- 
tures and differences from typical pre-COVID-19 ARDS. Med J Aust . Published online June 22, 2020. doi: $10.5694 / \mathrm{mja} 2.50674$

3. The ARDS Definition Task Force*. Acute Respiratory Distress Syndrome: The Berlin Definition. JAMA . 2012;307(23):2526-2533. doi:10.1001/jama.2012.5669

4. Kalil AC, Thomas PG. Influenza virus-related critical illness: pathophysiology and epidemiology. Critical Care . 2019;23(1):258. doi:10.1186/s13054-019-2539-x

5. Rozencwajg S, Bréchot N, Schmidt M, et al. Co-infection with influenza-associated acute respiratory distress syndrome requiring extracorporeal membrane oxygenation. Int J Antimicrob Agents . 2018;51(3):427433. doi:10.1016/j.ijantimicag.2017.11.005

6. Grasselli G, Tonetti T, Protti A, et al. Pathophysiology of COVID-19-associated acute respiratory distress syndrome: a multicentre prospective observational study. The Lancet Respiratory Medicine . Published online August 2020:S2213260020303702. doi:10.1016/S2213-2600(20)30370-2

7. Bartlett RH, Ogino MT, Brodie D, et al. Initial ELSO Guidance Document: ECMO for COVID-19 Patients with Severe Cardiopulmonary Failure. ASAIO J . 2020;66(5):472-474. doi:10.1097/MAT.0000000000001173

8. Clinical management of COVID-19. Accessed November 12, 2020. https://www.who.int/publicationsdetail-redirect/clinical-management-of-covid-19

9. Alhazzani W, Møller MH, Arabi YM, et al. Surviving Sepsis Campaign: guidelines on the management of critically ill adults with Coronavirus Disease 2019 (COVID-19). Intensive Care Med . Published online March 28, 2020:1-34. doi:10.1007/s00134-020-06022-5

10. Savarimuthu S, BinSaeid J, Harky A. The role of ECMO in COVID-19: Can it provide rescue therapy in those who are critically ill? Journal of Cardiac Surgery . 2020;35(6):1298-1301. doi:10.1111/jocs.14635

11. Sidebotham D, McGeorge A, McGuinness S, Edwards M, Willcox T, Beca J. Extracorporeal Membrane Oxygenation for Treating Severe Cardiac and Respiratory Disease in Adults: Part 1-Overview of Extracorporeal Membrane Oxygenation. Journal of Cardiothoracic and Vascular Anesthesia . 2009;23(6):886-892. doi:10.1053/j.jvca.2009.08.006

12. Ratnani I, Tuazon D, Zainab A, Uddin F. The Role and Impact of Extracorporeal Membrane Oxygenation in Critical Care. Methodist Debakey Cardiovasc J . 2018;14(2):110-119. doi:10.14797/mdcj-14-2-110

13. Munshi L, Walkey A, Goligher E, Pham T, Uleryk EM, Fan E. Venovenous extracorporeal membrane oxygenation for acute respiratory distress syndrome: a systematic review and meta-analysis. Lancet Respir Med . 2019;7(2):163-172. doi:10.1016/S2213-2600(18)30452-1

14. Brodie D, Bacchetta M. Extracorporeal Membrane Oxygenation for ARDS in Adults. New England Journal of Medicine . 2011;365(20):1905-1914. doi:10.1056/NEJMct1103720

15. Combes A, Hajage D, Capellier G, et al. Extracorporeal Membrane Oxygenation for Severe Acute Respiratory Distress Syndrome. N Engl J Med . 2018;378(21):1965-1975. doi:10.1056/NEJMoa1800385

16. Schmidt M, Pham T, Arcadipane A, et al. Mechanical Ventilation Management during Extracorporeal Membrane Oxygenation for Acute Respiratory Distress Syndrome. An International Multicenter Prospective Cohort. Am J Respir Crit Care Med . 2019;200(8):1002-1012. doi:10.1164/rccm.201806-1094OC

17. Sukhal S, Sethi J, Ganesh M, Villablanca PA, Malhotra AK, Ramakrishna H. Extracorporeal Membrane Oxygenation in Severe Influenza Infection with Respiratory Failure: A Systematic Review and Meta-analysis. Ann Card Anaesth . 2017;20(1):14-21. doi:10.4103/0971-9784.197820

18. Bagga S, Paluzzi DE, Chen CY, et al. Better Ventilator Settings Using a Computerized Clinical Tool. Respiratory Care . 2014;59(8):1172-1177. doi:10.4187/respcare.02223 
19. Hsu J, Santesso N, Mustafa R, et al. Antivirals for Treatment of Influenza. Ann Intern Med . 2012;156(7):512-524. doi:10.7326/0003-4819-156-7-201204030-00411

20. The effect of corticosteroids on mortality of patients with influenza pneumonia: a systematic review and meta-analysis | Critical Care | Full Text. Accessed April 20, 2021. https://ccforum.biomedcentral.com/articles/10.1186/s13054-019-2395-8

21. Consortium WST. Repurposed Antiviral Drugs for Covid-19 - Interim WHO Solidarity Trial Results. New England Journal of Medicine . Published online December 2, 2020. doi:10.1056/NEJMoa2023184

22. Li M, Yoo EJ, Baram M, et al. Tocilizumab in the Management of COVID-19: A Preliminary Report. The American Journal of the Medical Sciences . 2021;361(2):208-215. doi:10.1016/j.amjms.2020.11.005

23. Barbaro RP, MacLaren G, Boonstra PS, et al. Extracorporeal membrane oxygenation support in COVID-19: an international cohort study of the Extracorporeal Life Support Organization registry. The Lancet . 2020;396(10257):1071-1078. doi:10.1016/S0140-6736(20)32008-0

24. Schmidt M, Hajage D, Lebreton G, et al. Extracorporeal membrane oxygenation for severe acute respiratory distress syndrome associated with COVID-19: a retrospective cohort study. The Lancet Respiratory Medicine . 2020;8(11):1121-1131. doi:10.1016/S2213-2600(20)30328-3

25. Haiduc AA, Alom S, Melamed N, Harky A. Role of extracorporeal membrane oxygenation in COVID-19: A systematic review. Journal of Cardiac Surgery . 2020;35(10):2679-2687. doi:https://doi.org/10.1111/jocs.14879

26. Shaheen A, Tanaka D, Cavarocchi NC, Hirose H. Veno-Venous Extracorporeal Membrane Oxygenation (V V ECMO): Indications, Preprocedural Considerations, and Technique. J Card Surg . 2016;31(4):248-252. doi:10.1111/jocs. 12690

27. Sultanian P, Lundgren P, Stromsoe A, et al. Cardiac arrest in COVID-19: characteristics and outcomes of in- and out-of-hospital cardiac arrest. A report from the Swedish Registry for Cardiopulmonary Resuscitation. European Heart Journal . 2021;42(11):1094-1106. doi:10.1093/eurheartj/ehaa1067

28. Hirose H, Pitcher HT, Baram M, Cavarocchi NC. Issues in the Intensive Care Unit for Patients with Extracorporeal Membrane Oxygenation. Crit Care Clin . 2017;33(4):855-862. doi:10.1016/j.ccc.2017.06.007

29. Awsare B, Herman J, Baram M. Management Strategies for Severe Respiratory Failure: As Extracorporeal Membrane Oxygenation Is Being Considered. Critical Care Clinics . 2017;33(4):795-811. doi:10.1016/j.ccc.2017.06.003

30. Schmidt M, Stewart C, Bailey M, et al. Mechanical Ventilation Management During Extracorporeal Membrane Oxygenation for Acute Respiratory Distress Syndrome: A Retrospective International Multicenter Study*. Critical Care Medicine . 2015;43(3):654-664. doi:10.1097/CCM.0000000000000753

31. Blazoski C, Baram M, Hirose H. Outcomes of extracorporeal membrane oxygenation in acute respiratory distress syndrome due to COVID-19: The lessons learned from the first wave of COVID-19. Journal of Cardiac Surgery . n/a(n/a). doi:https://doi.org/10.1111/jocs.15512

32. Acosta MAT, Singer BD. Pathogenesis of COVID-19-induced ARDS: implications for an ageing population. European Respiratory Journal . 2020;56(3). doi:10.1183/13993003.02049-2020

33. Cates J, Lucero-Obusan C, Dahl RM, et al. Risk for In-Hospital Complications Associated with COVID19 and Influenza - Veterans Health Administration, United States, October 1, 2018-May 31, 2020. MMWR Morb Mortal Wkly Rep . 2020;69(42):1528-1534. doi:10.15585/mmwr.mm6942e3

34. Wu C-P, Adhi F, Highland K. Recognition and management of respiratory co-infection and secondary bacterial pneumonia in patients with COVID-19. CCJM . 2020;87(11):659-663. doi:10.3949/ccjm.87a.ccc015 
35. Dudoignon E, Camelena F, Deniau B, et al. Bacterial Pneumonia in COVID-19 Critically Ill Patients: A Case Series. Clinical Infectious Diseases . 2021;72(5):905-906. doi:10.1093/cid/ciaa762

36. Sharifipour E, Shams S, Esmkhani M, et al. Evaluation of bacterial co-infections of the respiratory tract in COVID-19 patients admitted to ICU. BMC Infectious Diseases . 2020;20(1):646. doi:10.1186/s12879-02005374-z

37. Vaillancourt M, Jorth P. The Unrecognized Threat of Secondary Bacterial Infections with COVID-19. mBio . 2020;11(4). doi:10.1128/mBio.01806-20

38. RECOVERY Collaborative Group, Horby P, Lim WS, et al. Dexamethasone in Hospitalized Patients with Covid-19 - Preliminary Report. N Engl J Med . Published online July 17, 2020. doi:10.1056/NEJMoa2021436

39. Guaraldi G, Meschiari M, Cozzi-Lepri A, et al. Tocilizumab in patients with severe COVID-19: a retrospective cohort study. The Lancet Rheumatology . 2020;2(8):e474-e484. doi:10.1016/S2665-9913(20)30173-9
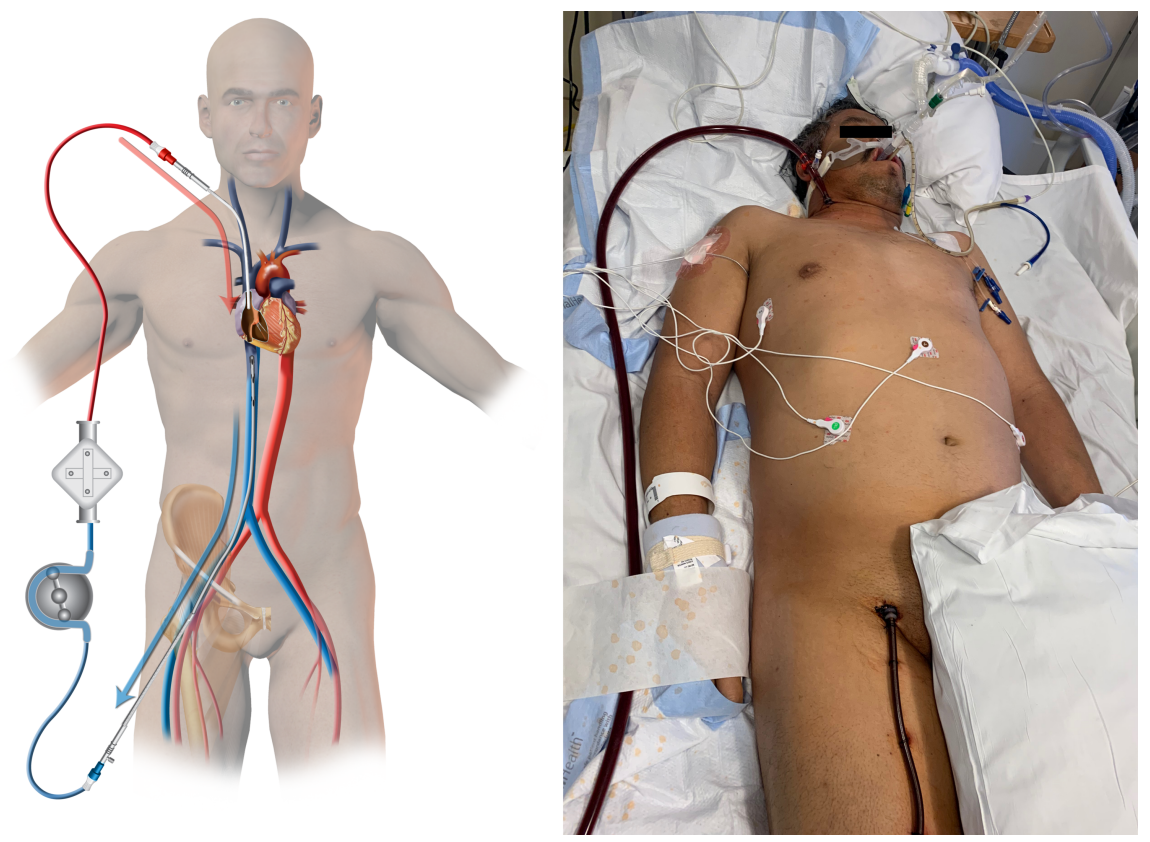